\title{
CXCR4 uses STAT3-mediated slug expression to maintain radioresistance of non-small cell lung cancer cells: emerges as a potential prognostic biomarker for lung cancer
}

\author{
Jeong-Yub Kim', Hee-Jin Kim ${ }^{1,2}$, Chan-Woong Jung ${ }^{1,3}$, Tae Sup Lee $\mathbb{D}^{4}$, Eun Ho Kim ${ }^{5}$ and Myung-Jin Park (1)
}

\begin{abstract}
Lung cancer is one of the most common reasons for cancer-induced mortality across the globe, despite major advancements in the treatment strategies including radiotherapy and chemotherapy. Existing reports suggest that CXCR4 is frequently expressed by malignant tumor and is imperative for vascularization, tumor growth, cell migration, and metastasis pertaining to poor prognosis. In this study, we infer that CXCR4 confers resistance to ionizing radiation (IR) in nonsmall cell lung cancer (NSCLC) cells. Further, on the basis of colony forming ability, one finds that drugresistant A549/GR cells with improved CXCR4 expression exhibited more resistance to IR than A549 cells evidenced along with a reduction in the formation of $\mathrm{Y}-\mathrm{H} 2 \mathrm{AX}$ foci after IR. Transfection of shRNA against CXCR4 or treatment of pharmacological inhibitor (AMD3100) both led to sensitization of A549/GR cells towards IR. Conversely, the overexpression of CXCR4 in A549 and H460 cell lines was found to improve clonogenic survival, and reduce the formation of $\mathrm{Y}$-H2AX foci after IR. CXCR4 expression was further correlated with STAT3 activation, and suppression of STAT3 activity with siSTAT3 or a specific inhibitor (WP1066) significantly stymied the colony-forming ability and increased $\mathrm{Y}$-H2AX foci formation in A549/GR cells, indicating that CXCR4-mediated STAT3 signaling plays an important role for IR resistance in NSCLC cells. Finally, CXCR4/STAT3 signaling was mediated with the upregulation of Slug and downregulation of the same with siRNA, which heightened IR sensitivity in NSCLC cells. Our data collectively suggests that CXCR4/STAT3/Slug axis is paramount for IR resistance of NSCLC cells, and can be regarded as a therapeutic target to enhance the IR sensitivity of this devastating cancer.
\end{abstract}

\section{Introduction}

With a high death burden across the globe, lung cancer has emerged as a major healthcare problem. Small cell lung cancer (SCLC) and nonsmall cell lung cancer (NSCLC) account for up to $87 \%$ of lung cancer cases, thus constituting most frequent types of cancers ${ }^{1}$. More

\footnotetext{
Correspondence: Eun Ho Kim (eh140149@cu.ac.kr) or Myung Jin Park (mjpark@kirams.re.kr)

${ }^{1}$ Radiation Therapeutics Development Team, Division of Radiation Cancer Science, Korea Institute of Radiological and Medical Sciences, Seoul, Korea

${ }^{2}$ School of Biomedical Science, Korea University, Seoul, Korea

Full list of author information is available at the end of the article

These authors contributed equally: Jeong-Yub Kim, Hee-Jin Kim

Edited by S. Tait
}

specifically, a $15 \%$ survival rate is seen in NSCLC patients. Despite employing numerous interventions like chemotherapy and radiotherapy, no such significant improvement is marked in the survival rate of the patients. This indicates a vast knowledge gap on the response of condition to various interventions and treatments as along with its tumorigenesis ${ }^{2}$. While chemotherapy remains the preferred option for treatment of NSCLCs with the exception of surgery; radiotherapy is the secondary option that continues to be one of the main treatment modality for those with independent NSCLC or with another form of treatment such as chemotherapy ${ }^{3}$. Notwithstanding the progress in radiation techniques, the

\section{(c) The Author(s) 2021}

\footnotetext{
(c) Open Access This article is licensed under a Creative Commons Attribution 4.0 International License, which permits use, sharing, adaptation, distribution and reproduction cc) in any medium or format, as long as you give appropriate credit to the original author(s) and the source, provide a link to the Creative Commons license, and indicate if changes were made. The images or other third party material in this article are included in the article's Creative Commons license, unless indicated otherwise in a credit line to the material. If material is not included in the article's Creative Commons license and your intended use is not permitted by statutory regulation or exceeds the permitted use, you will need to obtain permission directly from the copyright holder. To view a copy of this license, visit http://creativecommons.org/licenses/by/4.0/.
} 
survival rate of patients still needs improvement. A considerable number of patients are known to witness either local-regional recurrence or new cases of primary cancer after radiotherapy. For this reason it is necessary to develop radiotherapeutic strategies by focusing the crucial mechanism(s) for lung cancer's radioresistance so as to improve the treatment outcomes.

The existence of cancer stem cells (CSCs) that trigger tumor heterogeneity are considered to be one of the most common reasons behind therapeutic failure after drastic radiotherapy and chemotherapy. Initially, lung-cancerrelated CSCs (LCSCs) were found in the subpopulation of cells with CD133 on their cell surface extracted from the tissues of patients, both in SCLC and NSCLC cases ${ }^{4}$. LCSCs expressed exaggerated levels of embryonic stem cell factor, Oct4, and Sox2, followed by drug pumping protein, $A B C G 2$, all these factors are deemed to be responsible for self-renewal and chemoresistance, respectively ${ }^{5,6}$. Furthermore, aldehyde dehydrogenasepositive lung cancer cells and urokinase plasminogen activator receptor-positive also exhibit features of $\mathrm{CSCs}^{7}$. According to various reports, lung cancer cells that are resistant to ionizing radiation (IR) and drugs which exhibit CSCs characteristics are able to express several epithelial-mesenchymal transition makers and $\mathrm{CSCs}^{8}$. It is for this reason that it is necessary to target LCSCs in order to augment the clinical outcomes of lung cancer patients.

CXCR4 (chemokine (C-X-C motif) receptor 4) is a receptor for a chemokine stromal-derived growth factor$1 \alpha$ (SDF-1 $\alpha$ ), also known as CXCL12. Existing reports suggest that malignant tumors widely express CXCR4, which a paramount factor responsible for rapid growth, metastasis, and vascularization, along with poor prognosis. CXCR $4 / \mathrm{SDF}-1 \alpha$ axis seemingly pertains to the NSCLC's metastatic potential'. Activation of CXCR4 signaling increases the migration and invasion of NSCLC cells and blockade of this signaling reverses the effect in vitro and suppresses metastatic activity of these cells in vivo using neutralizing antibody ${ }^{10}$. CXCR4 is also called CSC marker since CSCs have the high level of CXCR4 expression on their surface ${ }^{11,12}$. Our previous study also found that CXCR4 is a functional LCSC marker for maintenance of stemness and tumorigenesis in NSCLC cells ${ }^{13}$. By employing transfection with antisense nucleotide for CXCR4 or blockade of CXCR4/SDF- $1 \alpha$ axis with pharmacological inhibitor or neutralizing antibody, we concluded CXCR4 as a crucial factor for the maintenance of stemness, tumorigenesis and IR resistance in NSCLC cells ${ }^{13}$.

In the current study, we aim to discern the molecular mechanism of CXCR4-mediated IR resistance in NSCLC cells. Our results showed that CXCR4 signaling was proven to be crucial for IR-induced DNA damage repair in NSCLC cells. CXCR4 signaled to STAT3/Slug axis thereby reduced DNA damage caused by IR and increased clonogenic survival in NSCLC cells. Therefore, our results offer compelling evidence that targeting CXCR4 and STAT3/Slug signaling would be useful for enhancing IR sensitivity in NSCLC.

\section{Materials and methods \\ Antibodies and reagents}

Antibody against $\mathrm{H} 2 \mathrm{AX}$ and $\gamma-\mathrm{H} 2 \mathrm{AX}$ were obtained from MilliporeSigma (Burlington, MA). CXCR4 was purchased from LS Bioscience (Seattle, WA). Antibody such as p-STAT3 (S727) and $\beta$-actin were purchased from Santa Cruz Biotechnology (Santa Cruz, CA). p-STAT3 (Y705) and Slug were obtained from Cell Signaling Biotechnology (Denvers, MA). AMD3100 and WP1066 were obtained from Sigma-Aldrich (St. Louis, MO). SDF- $1 \alpha$ was purchased from R\&D systems (Minneapolis, $\mathrm{MN})$.

\section{Cell culture}

NSCLC cell lines (A549 and H460) were obtained from ATCC (Manassas, VA). A549/GR cells were established as described previous reports ${ }^{14}$. All cell lines were cultured in RPMI1640 (Welgene, Gyeongsan-si, Gyeongsangbukdo, Korea) supplemented with $2 \mathrm{mM}$ glutamine, $1 \mathrm{mM}$ sodium pyruvate, $100 \mathrm{U} / \mathrm{ml}$ penicillin, $100 \mu \mathrm{g} / \mathrm{ml}$ streptomycin (Welgene) and 10\% fetal bovine serum (FBS; GIBCO-Thermo Fisher Scientific, Waltham, MA) in a humidified incubator containing $5 \% \mathrm{CO}_{2}$ at $37^{\circ} \mathrm{C}$.

\section{Knockdown or overexpression of CXCR4}

For knockdown of CXCR, pGFP-C-shLenti and pGFPC-shLenti CXCR4 vector were obtained from Origene (Rockville, MD). For overexpression of CXCR4, pQCXIP retrovirus vector was obtained from Takara Bio USA (Mountain View, CA) and CXCR4 was subcloned into the vector. Production of viruses was done by transfection of $293 \mathrm{~T}$ cells with viral vectors and packaging mix using TransIT-X2 (Mirus Bio LLC, Madison, WI). After $48 \mathrm{~h}$ of transfection, viral supernatants were collected, filtered, and stored at deep freezer with polybrene $(8 \mu \mathrm{g} / \mathrm{ml}$; Sigma-Aldrich) until use.

\section{Transfection of small interfering RNA (siRNA)}

Cells were transfected with a pool of siRNAs against CXCR4: 5'-AUCACGUAAAGCUAGAAA- $3^{\prime}$ and $5^{\prime}$-GGG AUCAUUUCUAGCUUU-3'; STAT3: 5'-UCCAGUUUC UUAAUUUGUUGACGGGUU- ${ }^{\prime}$ and $5^{\prime}$ - GGCCAUGAA CUUGACAAUAUCUGCUUU-3', Slug: 5'-GAGAGAU UAUCUAUGCAUAAACAGCUU- $3^{\prime}$ and $5^{\prime}$-ACCAGCA UUUCUAUACCACUUUGGGUU-3' (Integrated DNA Technologies, Coralville, IA) at a final concentration of $20 \mathrm{nM}$ using G-fectin (Genolution, Seoul, Korea). 
Semiquantitative reverse transcriptase-polymerase chain reaction (sqRT-PCR) and quantitative RT-PCR (qRT-PCR)

High-quality total RNA was isolated from cells by using TRIsure (BIOLINE, London, UK) as described in the manufacturer's protocol. Complimentary DNA was synthesized using the cDNA synthesis kit (BIOLINE). Oligonucleotide primer sequences for sqRT-PCR used were as follows: CXCR4 forward: 5'-AATCTTCCTGCCCACC ATCT-3', CXCR4 reverse: 5'-GACGCCAACATAGACC ACCT-3'; STAT3 forward: 5'-TAATGAAAAGTGCCT TTGTGG-3', STAT3 reverse: 5'-TGACCAGCAACCTG ACTTTAG-3', Slug forward: 5'-TGTGACAAGGAAT ATGTGAGCC-3', Slug reverse: 5'-TGAGCCCTCAGATT TGACCTG-3', GAPDH forward: 5'-TGGTGAAGGTCG GTGTGAAC-3', GAPDH reverse: 5'-TTCCCATTCTC AGCCTTGAC-3'.

cDNAs were analyzed by qPCR (CFX96 Touch $^{\text {TM }}$ Realtime detection system, Biorad, Hercules, CA) using SYBR (BIOLINE). Samples were assayed in triplicate for each gene and relative expression was calculated by the $\Delta \Delta \mathrm{Ct}$ method (Applied Biosystems, Foster city, CA). Oligonucleotide primer sequences for qRT-PCR used were as follows: CXCR4 forward: 5'-TCAACCTCTACA GCAGCGTTCTCTT-3', CXCR4 reverse: 5'-TGTTG GTGGCGTGGACAAT- $3^{\prime}$. The qRT-PCR primers of STAT3 and Slug were the same as sqRT-PCR primers.

\section{Western blot analysis}

Cells were lysed in RIPA buffer (50 mM Tris- $\mathrm{HCl}(\mathrm{pH}$ 7.4), $100 \mathrm{mM} \mathrm{NaCl}, 5 \mathrm{mM}$ EDTA, $0.5 \%$ Nonidet P-40, phosphatase inhibitor cocktail setII (MilliporeSigma), and a protease inhibitor cocktail tablet (Roche, Basel, Switzerland). The protein contents were determined using the protein assay reagent (Bio-Rad, Hercules, CA). The proteins were separated using 8-12\% SDS-PAGE gels and transferred to a nitrocellulose membrane. The membranes were blocked with $5 \%$ skim milk in TBST $(20 \mathrm{mmol} / \mathrm{l}$ Tris- $\mathrm{HCl}(\mathrm{pH}$ 7.6), $137 \mathrm{mmol} / \mathrm{lNaCl}$ and $0.01 \%$ Tween-20) for $1 \mathrm{~h}$ at room temperature (RT) and then incubated with the indicated primary antibodies overnight at $4{ }^{\circ} \mathrm{C}$ with gentle shaking. After extensive washing with TBST, the membrane was developed with a peroxidase-conjugated secondary antibody for $2 \mathrm{~h}$ at RT. After washing three times with TBST for $10 \mathrm{~min}$, membranes were visualized by enhanced chemiluminescence (Amersham, Pittsburgh, PA) according to the manufacturer's protocol.

\section{Irradiation and clonogenic assay}

For measuring IR sensitivity of NSCLC cells, cells were seeded in 6-well plates $\left(1 \times 10^{3}\right.$ cells $\left./ \mathrm{ml}\right)$ and exposed to $\gamma$-rays from a ${ }^{137} \mathrm{Cs} \gamma$-ray source (BIOBEAM8000, $2.6 \mathrm{~Gy} /$ min, Gamma-service Medical GmbH, Leipzig, Germany) at the indicated dose rate. After 7 days of incubation, spheres were attached by adding $10 \%$ FBS, washed with PBS, and stained with $0.05 \%$ crystal violet dissolved in $20 \%$ methanol. After washing three times with distilled water, colonies were counted.

\section{Immunocytochemistry (ICC) and $\mathrm{p}-\mathrm{H} 2 \mathrm{AX}$ foci assay}

Cells were seeded in a chamber slide with DMEM supplemented with $10 \%$ FBS. The next day, the cells were fixed with $4 \%$ paraformaldehyde for $10 \mathrm{~min}$ and washed with PBS three times. Then, the cells were incubated in blocking solution (5\% BSA and 0.5\% Triton X-100 in PBS) for $1 \mathrm{~h}$ at RT. The cells were stained with primary antibodies $(\gamma-\mathrm{H} 2 \mathrm{AX})$ in blocking solution $(1: 100)$ for $2 \mathrm{~h}$ and washed with PBS three times. Then, the cells were incubated with Alexa Fluor 594-labeled goat anti-mouse (Bethyl Laboratories, Montgomery, TX) secondary antibodies (1:1000) for $1 \mathrm{~h}$. Nuclei were stained with DAPI and stained cells were viewed under a confocal laserscanning microscope. $\gamma$-H2AX foci were determined in at least 50 cells.

\section{Immunohistochemistry (IHC)}

IHC was performed to assess the expression of CXCR4, p-STAT3 (S705), and Slug in tumor xenograft sections and tissue array slide purchased from US Biomax (LC1921b.; Rockville, MD, USA). IHC was performed using antibodies against CXCR4, p-STAT3 (S705), and Slug (1:100), and secondary antibodies conjugated with the fluorescent dyes Alexa 488 or Alexa 555 (1:400; Jackson Labs). Tumor sections positively stained areas were evaluated with ImageJ software (http://imagej.net/).

\section{Cell cycle analysis}

Cells were resuspended in 100\% cold $\mathrm{EtOH}$ and chilled on ice overnight. The cells were then washed with PBS and stained with PBS containing $50 \mu \mathrm{g} / \mathrm{ml} \mathrm{PI}, 10 \mu \mathrm{g} / \mathrm{ml}$ ribonuclease $\mathrm{A}$, and $0.05 \%$ Triton $\mathrm{X}-100$ for $40 \mathrm{~min}$ in the dark. After centrifugation, cells were resuspended in PBS. Flow cytometry analysis was performed on FACS Calibur (Becton Dickinson).

\section{Animal study}

All of the experiments were conducted using protocols and conditions approved by the Institutional Animal Care and Use Committee in KIRAMS. Athymic BALB/c female mice (Nara Biotech, Seoul, Korea) were subcutaneously injected with A549/GR cells $\left(5 \times 10^{6}\right.$ cells in PBS) in right thigh. A549/GR tumor bearing mice were randomized into four groups $(n=6-7)$. Treatment was performed with AMD3100 alone, irradiation alone, AMD3100 combined with irradiation, or control. Treatment with AMD3100 was started at -1 day before irradiation and maintained for 7 days. Control or AMD3100 treated group implanted with 7 days $0.5 \mu \mathrm{l} / \mathrm{h}$ osmotic pump 
(Alzet, Cupertino, CA) loaded with saline or $25 \mathrm{mg} / \mathrm{ml}$ of AMD3100 before one day irradiation. Irradiation was performed with X-ray unit operated at $260 \mathrm{kVp}$ with a dose rate of $2 \mathrm{~Gy} / \mathrm{min}$ (10 $\mathrm{mA}$ with added filtration of $2 \mathrm{~mm}$ copper, distance from X-ray source to the target of $41 \mathrm{~cm})$. Tumor volume $\left(\mathrm{mm}^{3}\right)$ was calculated using following formula: long diameter $\times(\text { short diameter })^{2} \times 0.5$. Tumor growth rate was compared by using Tumor volume doubling time (TDT). Body weight of the mice was monitored during all the treatments. No significant losses in body weight (less than 10\%) were observed.

\section{Preparation of ${ }^{64} \mathrm{Cu}$-AMD3100}

AMD3100 was purchased from Sigma-Aldrich, and ${ }^{64} \mathrm{Cu}$ was produced at KIRAMS by $50 \mathrm{MeV}$ cyclotron irradiation using methods reported ${ }^{15} \cdot\left[{ }^{64} \mathrm{Cu}\right] \mathrm{AMD} 3100$ was prepared according to literature method ${ }^{16}$. Briefly, $500 \mathrm{ng}$ of AMD3100 were added to $37 \mathrm{MBq}$ of ${ }^{64} \mathrm{CuCl}_{2}$ buffered with $1 \mathrm{M}$ sodium acetate buffer $(\mathrm{pH}$ 6.5) and incubated at $60^{\circ} \mathrm{C}$ for $40 \mathrm{~min}$. Quality control was performed by instant thin layer chromatography-silica gel (ITLC-sg, Pall, USA) with a mobile phase of $20 \mathrm{mM}$ citrate buffer pH 5 with $50 \mathrm{mM}$ EDTA.

\section{Receptor binding assay of $\left[{ }^{64} \mathrm{Cu}\right] \mathrm{AMD} 3100$}

A549 and A549/GR cells were seeded in 6-well plate $\left(5 \times 10^{5}\right.$ cells/well $)$ and incubated with $18.5 \mathrm{kBq} / \mathrm{ml}$ of $\left[{ }^{64} \mathrm{Cu}\right]$ AMD3100 for $1 \mathrm{~h}$ in incubation buffer (Hank's balanced salt solution with $0.25 \%$ BSA). After incubation, cells were triple washed with incubation buffer and detached, cell bound radioactivity was determined in a gamma counter (Wizard 2480, Perkin-Elmer, USA). Radioactivity value was converted to percentage of added radioactivity dose (\%AD) per million cells. Experiments were performed in triplicate.

\section{PET/CT imaging of $\left[{ }^{64} \mathrm{Cu}\right] \mathrm{AMD} 3100$}

Athymic BALB/c female mice (Nara Biotech) were subcutaneously injected with A549 or A549/GR cells (5 × $10^{6}$ cell in PBS) in right flank. After 24 days, PET/CT images were obtained by using Inveon PET/CT system (Simens Preclinical Solution, Germany). Before the injection of radiotracer, mice were anesthesized with $3 \%$ isofluorane and maintained under $2 \%$ isofluorane. Whole body imaging was acquired for $30 \mathrm{~min}$ static scan at 90 min post injection of $\left[{ }^{64} \mathrm{Cu}\right]$ AMD3100 (11.1 MBq). The percentage of injected dose per gram $(\% \mathrm{ID} / \mathrm{g})$ value was calculated previously reported method ${ }^{17}$. Data were analyzed using Inveon Research Workplace software.

\section{Statistics analysis}

Data are presented as mean \pm SEM of at least three independent experiments. Differences were analyzed using the Student's $t$-test and were considered significant at ${ }^{*} P<0.05,{ }^{* *} P<0.01,{ }^{* * * *} P<0.005$. Statistical analysis and graphing were performed using the Microsoft Excel 2013 and GraphPad Prism 6.0 software (GraphPad software).

\section{Results \\ A549/GR cells are more resistant to IR than parent A549 cells}

In our previous report, it was shown that CXCR4+ cells are more resistant to IR as compared to CXCR4- cells in A549/GR cells ${ }^{13}$. To verify this phenomenon, we first examined whether A549/GR cells having high level of CXCR4 expression on their surface are more resistant to IR than A549 parent cells. In clonogenic assay, A549/GR cells exhibited high colony forming ability following IR exposure in contrast to A549 cells (Fig. 1A). IR may cause DNA damage along with checkpoint kinases activation, which could be detected by employing assays for examining the role in recruiting and phosphorylation of histone H2AX at ser-139 $(\gamma-\mathrm{H} 2 \mathrm{AX})^{18}$. As shown in Fig. 1B, Western blot analysis showed that expression of $\gamma-\mathrm{H} 2 \mathrm{AX}$ was lower in A549/GR cells than A549 cells after IR. ICC data revealed that IR-induced formation of $\gamma-\mathrm{H} 2 \mathrm{AX}$ foci started at 5 min and persisted upto $2 \mathrm{~h}$ in A549 cells. IR also induced a considerable increase of $\gamma-\mathrm{H} 2 \mathrm{AX}$ foci in A549/GR cells at five min, however, it was much lower than A549 cells and started to be resolved at 15 min time point and almost completely disappeared at an $\mathrm{h}$ after IR (Fig. 1C). In the cell cycle analysis, IR exposure led to a higher increase in the subG1 fractions. This indicates more cell deaths in A549 cells than in A549/GR cells (Supplemental Fig. 1A). Accordingly, Western blot analysis revealed that IR exposure caused a higher expression of cleaved caspase 3 (cCas3) and cleaved Parp (cParp) in A549 cells than in A549/GR cells (Supplemental Fig. 1b). These data suggest that A549/GR cells have higher activity of repairing DNA damage by IR evidenced by $\gamma-\mathrm{H} 2 \mathrm{AX}$ foci assay than A549 parent cells, which might lead to enhanced clonogenic survival of the latter after IR. To investigate the functional role of CXCR4 signaling in the survival activity of NSCLC cells from IR exposure, we treated the cells (A549, A549/GR, and H460) with SDF- $1 \alpha$, a ligand of CXCR4 and AMD3100, a specific antagonist of CXCR4 and performed clonogenic survival assays. As shown in Fig. 1D (left panel), pretreatment of SDF-1 $\alpha$ was found to improve clonogenic survival of A549/GR cells. Conversely, blockade of CXCR4 signaling with AMD3100 significantly suppressed colony-forming ability after IR in A549/GR cells (Fig. 1D, right panel). Clonogenic survival rate was also increased by SDF- $1 \alpha$ and decreased by AMD3100, respectively in A549 and H460 cells after IR, although it was not statistically significant (Supplemental Fig. 2A, B). These data collectively suggest that activation of CXCR4 signaling is important for NSCLC cells to survive from the IR-induced DNA damage. 


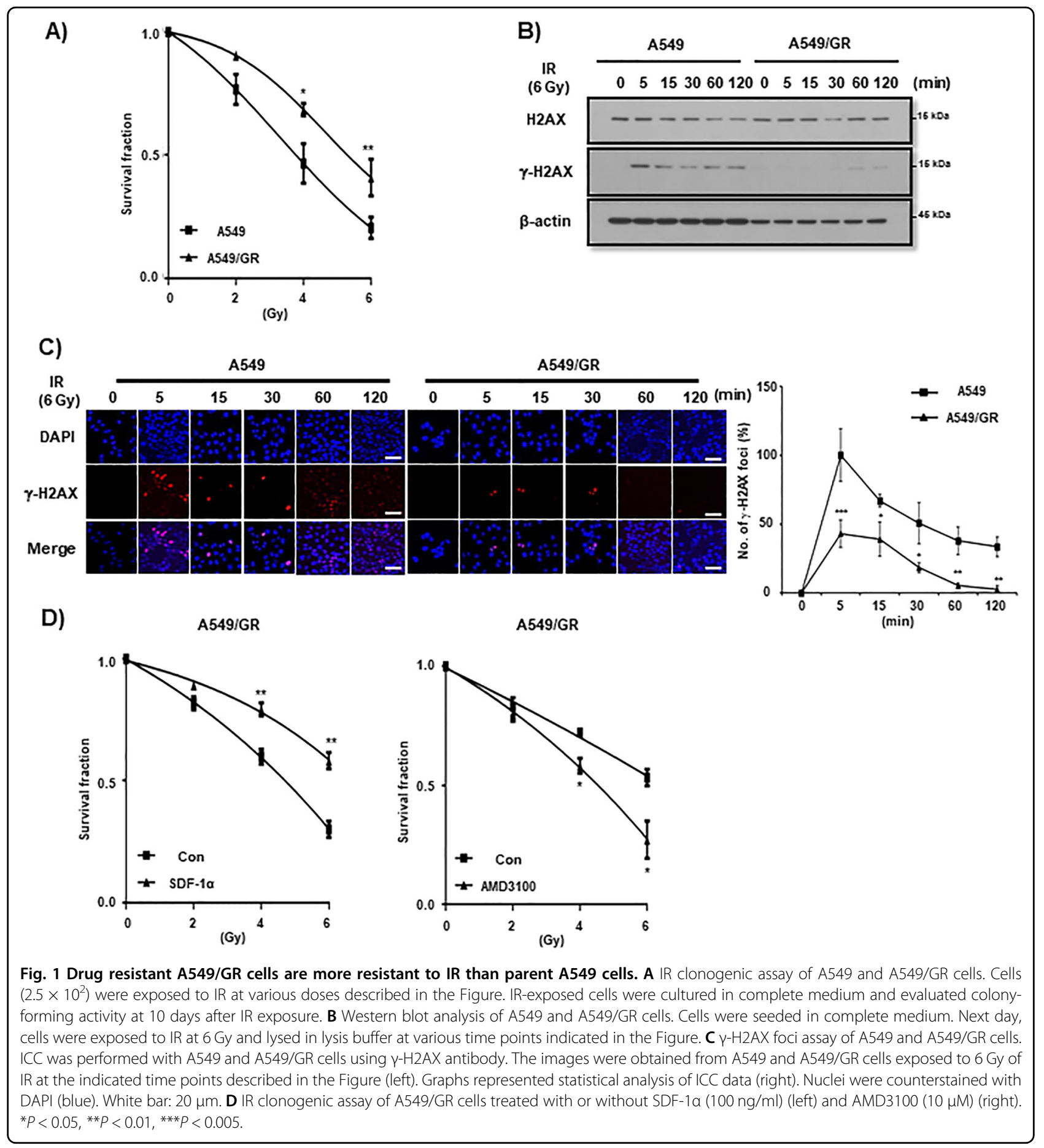

\section{CXCR4 knockdown sensitizes A549/GR cells to IR}

In order to ascertain the involvement of CXCR4 signaling in IR resistance of NSCLC cells, we performed the study dealing with the effects of loss of function of a CXCR4. To that end, we introduced shCXCR4 into A549/GR cells for knockdown the CXCR4 (Fig. 2A and Supplemental Fig. 3A). As shown Fig. 2B, knockdown of CXCR4 decreased clonogeinc survival activity of A549/GR cells to IR. Western blot analysis and $\gamma-\mathrm{H} 2 \mathrm{AX}$ foci assay also revealed that targeting CXCR4 enhanced the expression of $\gamma-\mathrm{H} 2 \mathrm{AX}$ (Fig. 2C), and caused the persistence of $\gamma-\mathrm{H} 2 \mathrm{AX}$ foci formation upto $2 \mathrm{~h}$ investigation after IR in these cells (Fig. 2D), respectively. Additionally, cell cycle and Western blot analysis revealed that CXCR4 knockdown increased sub-G1 fractions and cCas3 and cParp expression A549/GR cells (Supplemental Fig. 3B, C). 

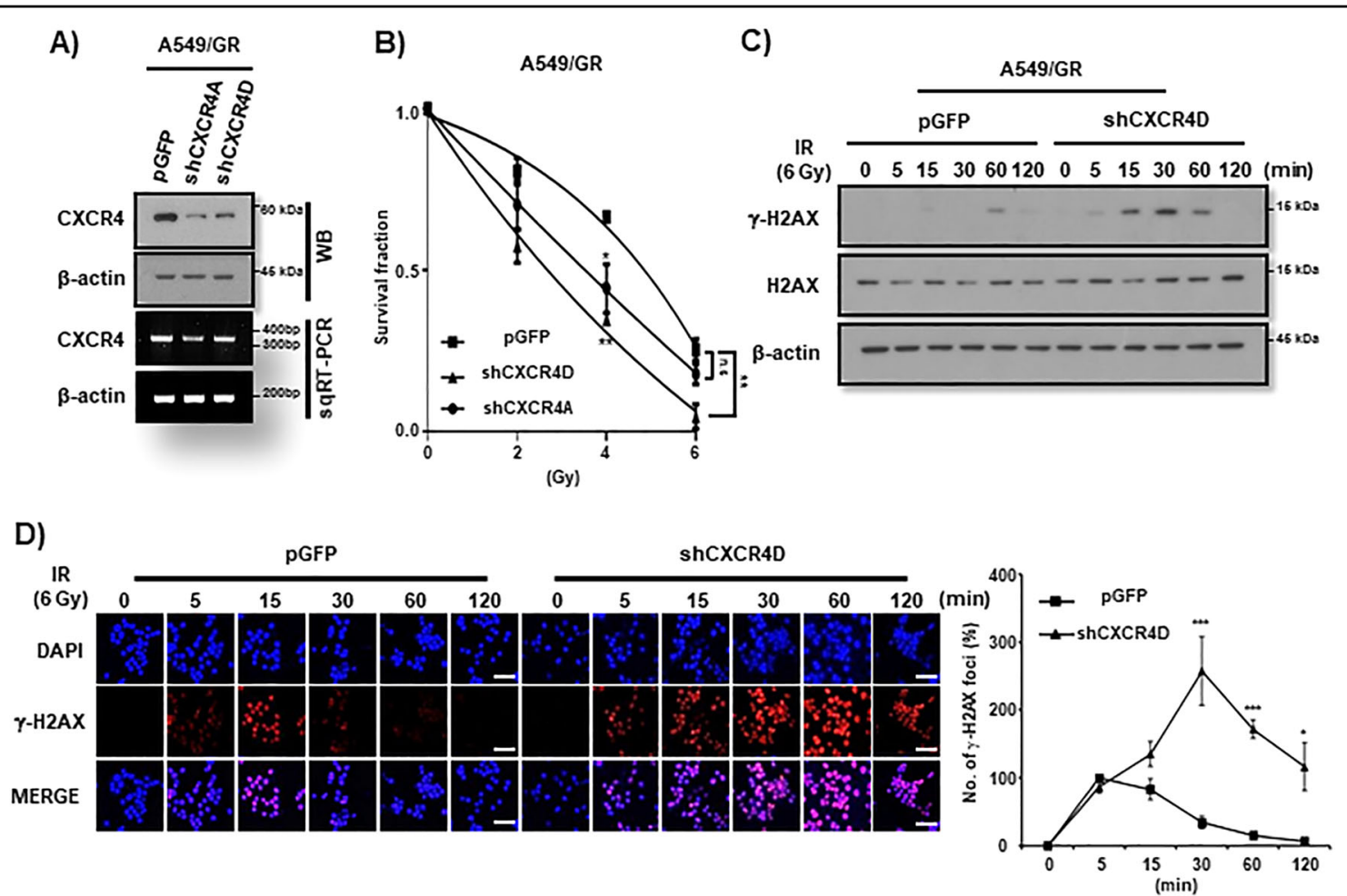

Fig. 2 Knockdown of CXCR4 decreases the survival of A549/GR cells to IR. A Western blot (WB, upper) and RT-PCR (lower) analysis of CXCR4 in A549/GR cells transfected with shControl (pGFP) or shCXCR4A and D. B IR clonogenic assay of A549/GR cells transfected with shCXCR4D. Cells (2.5 $\times$ $10^{2}$ ) were exposed to IR at various doses described in the Figure. IR-exposed cells were evaluated colony-forming activity at 10 days after IR. ${ }^{*} P<0.05$, ${ }^{*} P<0.01,{ }^{* *} P<0.005$. C Western blot analysis of A549/GR cells exposed to IR at 6 Gy at the indicated time points transfected with shControl (pGFP) or shCXCR4D. D $\gamma$-H2AX foci assay of A549/GR cells. ICC was performed with the cells transfected with shControl (pGFP) or shCXCR4D using $\gamma$-H2AX antibody after exposing to IR (6 Gy) at the indicated time points. Representative immunofluorescence images of A549/GR cells (left) and quantification of the assay (right). Nuclei were counterstained with DAPI (blue). White bar: $20 \mu \mathrm{m}$.

CXCR4 overexpression enhanced IR resistance in A549 and H460 NSCLC cells

Next, we examined the IR resistance effect of CXCR4 by the study of gain of function of CXCR4. Overexpression of CXCR4 evidenced by Western blot analysis and RT-PCR (Fig. 3A and Supplemental Fig. 4A) in A549 and H460 cells significantly increased IR resistance of these cells in clonogenic assay (Fig. 3B). $\gamma-\mathrm{H} 2 \mathrm{AX}$ foci assay also revealed enhanced foci resolution by CXCR4 expression in these cells (Fig. 3C). Western blot analysis clearly revealed that CXCR4 expression downregulated $\gamma-\mathrm{H} 2 \mathrm{AX}$ expression by IR after overexpression of CXCR4 (Fig. 3D). Cell cycle and Western blot analysis also indicated that overexpression of CXC4 suppressed the increase of subG1 fractions and cCas3 and cParp expression in A549 and H460 cells (Supplemental Fig. 4B-D).

\section{CXCR4-mediated STAT3 activation controls the IR resistance in NSCLC cells}

Previously, we found that CXCR4 signaled to STAT3 in order to maintain stemness in NSCLC cells ${ }^{13}$. Thus, we investigated the involvement of STAT3 in the CXCR4mediated IR resistance of NSCLC cells. Overexpression of CXCR4 stimulated STAT3 phosphorylation, but knockdown of CXCR4 constitutively decreased phosphorylation of STAT3 at the tyrosine 705 (pY-STAT3), but not at the serine 727 sites (pS-STAT3) in NSCLC cells (Fig. 4A). siRNA-mediated knockdown of STAT3 (Fig. 4B, left panel and Supplemental Fig. 5A) significantly reduced clonogenic survival of A549/GR cell after IR (Fig. 4B, right panel). In the $\gamma$-H2AX foci assay, treatment of si-STAT3 led to increase the foci formation, and delay the resolution time after IR in A549/GR cells (Fig. 4C). Western blot analysis also showed the upregulation of $\gamma$-H2AX expression upon siSTAT3 transfection (Fig. 4D). Upon IR exposure, knockdown of STAT3 enhanced subG1 fractions and cCas3 and cParp expression in A549/GR cells (Supplemental Fig. 5B, C). In addition, transfection of siSTAT3 significantly reduced clonogenic survival of CXCR4 overexpressed NSCLC cells (Fig. 4E, F). $\gamma$-H2AX foci assay demonstrated that treatment of siSTAT3 resulted in reduced foci formation after IR in CXCR4 


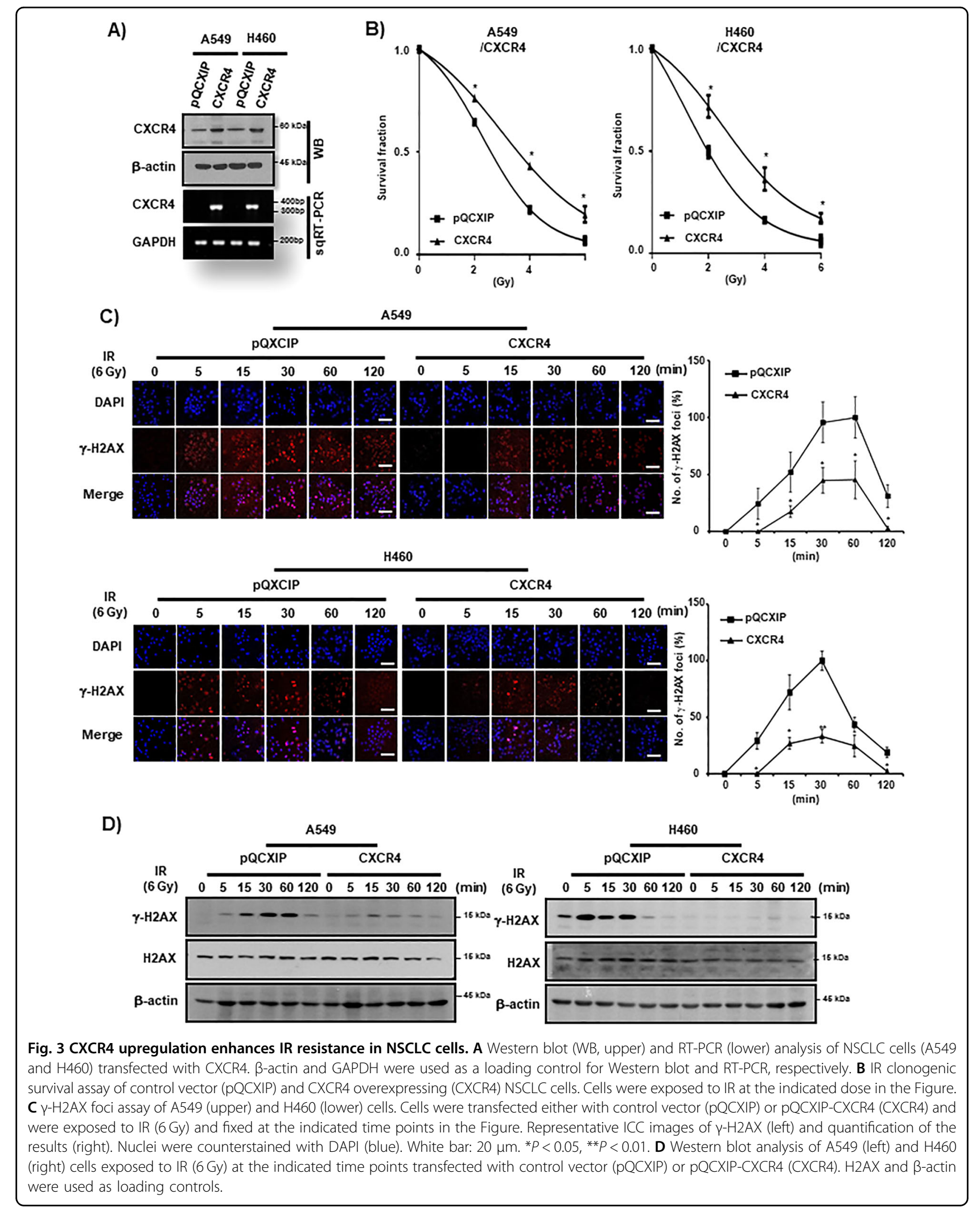




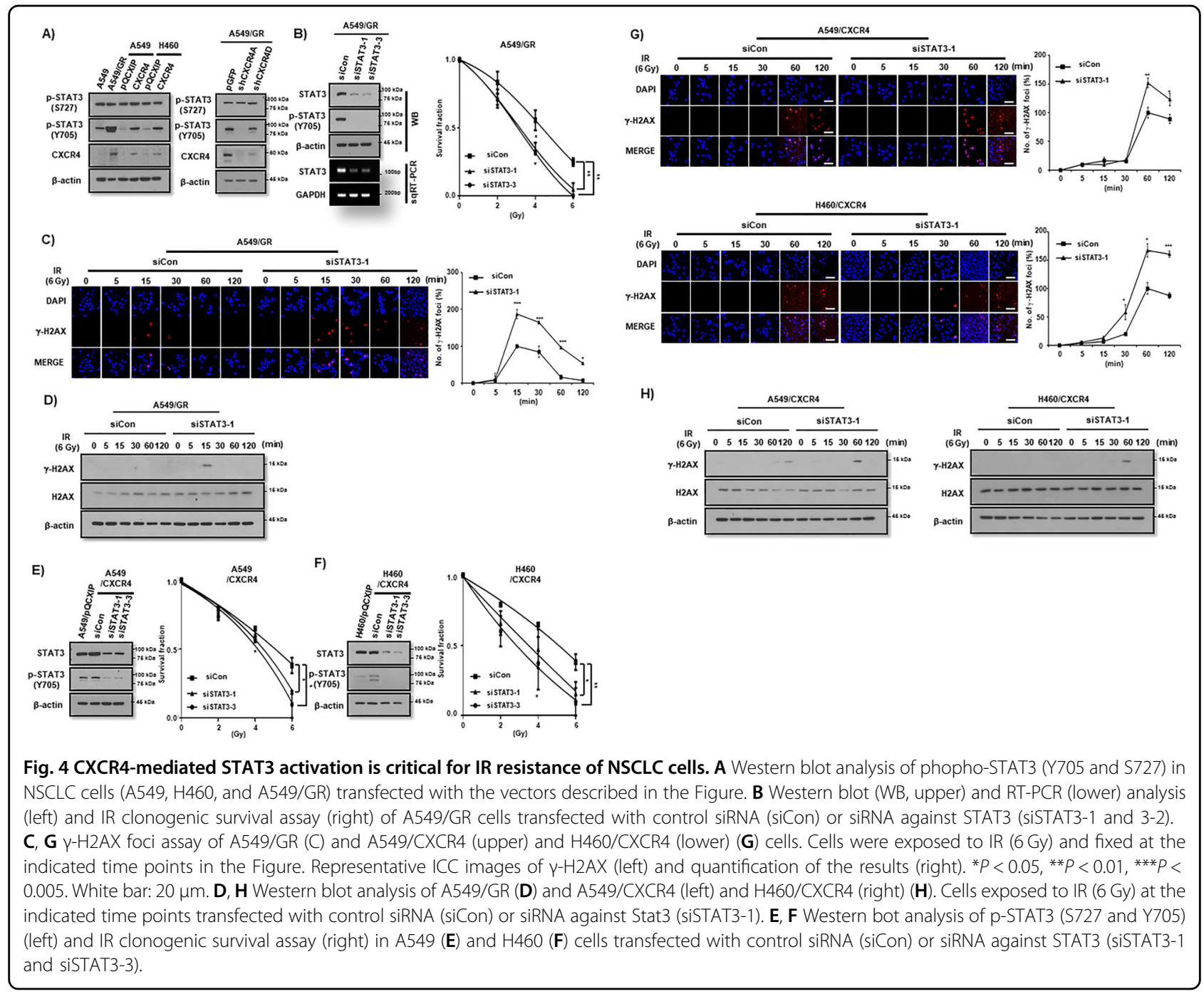

overexpressed A549 and H460 cells (Fig. 4G). Western blot analysis also showed elevated $\gamma-\mathrm{H} 2 \mathrm{AX}$ expression after transfection of siSTAT3 in these cells (Fig. 4H). Finally, pretreatment of WP1066, a specific inhibitor of STAT3 decreased clonogenic survival of CXCR4overexpressed NSCLC cells after IR (Supplemental Fig. 6). Taken together, IR stimulation of CXCR4 signals to STAT3 at the tyrosine 705 (pY-STAT3), which might be one of the major pathways for NSCLC cells to overcome IR-induced DNA damage.

\section{CXCR4/STAT3 signaling promotes IR resistance through Slug expression}

Next, we performed microarray to find downstream effector molecule for CXCR4/STAT3 signaling that is responsible for IR resistance in NSCLC cells. With the criteria of $P<0.01$ and $|\log 2(\mathrm{FC})| \geq 2,1231$ differentially expressed genes (DEGs) were identified when comparing A549/GR with A549, 678 DEGs were found when comparing A549/CXCR4 with control A549 and 298 DEGs when comparing H460/CXCR4 with H460. As presented in Fig. 5A, following construction of a Venn diagram, 17 DEGs were significantly differentially expressed among all three groups (Fig. 5A). Among these genes, we selected Snai2, also named Slug, as a possible effector molecule of CXCR4/STAT3 signaling, since several reports have shown the role of Slug in IR resistance of various cancer cells ${ }^{13-19}$. Western blot analysis revealed that Slug expression was upregulated in A549/GR cell line and CXCR4 overexpressing A549 and H460 cells in contrast to parent cells (Fig. 5B). Knockdown of CXCR4 suppressed Slug expression (Fig. 5C), whereas activation of CXCR4 signaling with SDF- $1 \alpha$ increased Slug expression in A549/GR cells (Fig. 5D and Supplemental Fig. 7A). In addition, pretreatment of AMD3100 also inhibited the Slug expression in A549/GR cells (Fig. 5E and Supplemental Fig. 7B). Transfection of siSTAT3 suppressed Slug expression in A549/GR and 


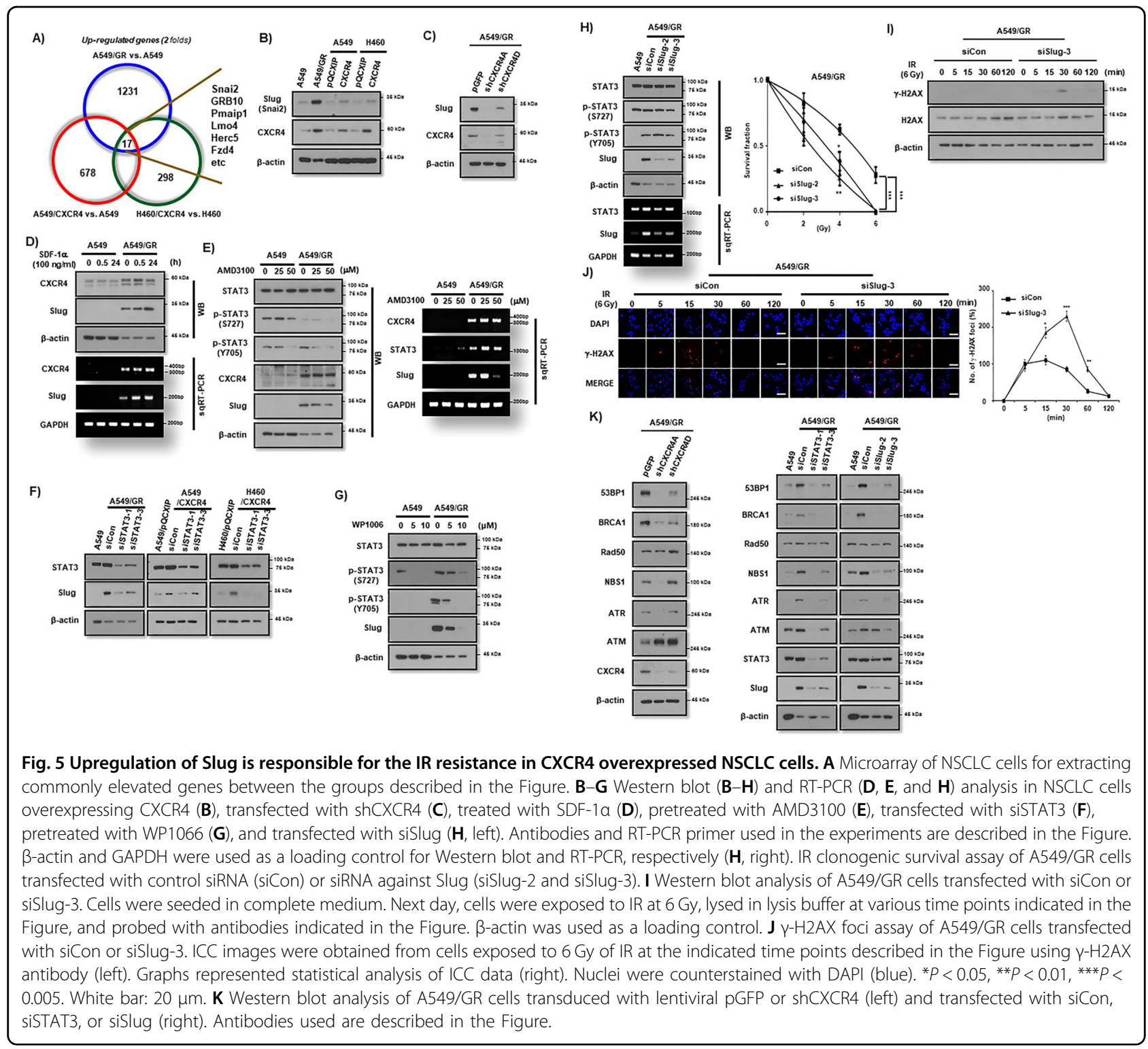

CXCR4 overexpressing A549 and H460 cells lines (Fig. 5F). Similarly, STAT3 inhibitor (WP1006) inhibited the expression of Slug in A549/GR cells (Fig. 5G). Importantly, siRNA-mediated suppression of Slug significantly decreased clonogenic survival (Fig. $5 \mathrm{H}$ and Supplemental Fig. 7C) and increased $\gamma$-H2AX expression (Fig, 5I) and foci (Fig. 5J) in A549/GR cells after IR. Also, Knockdown of Slug increased IR-induced subG1 fractions and cCas3 and cParp expression in A549/GR cells (Supplementary Fig. 7D, E). Finally, transfection of siCXCR4, siSTAT3, and siSlug significantly suppressed the expression of DNA repair-related proteins, including 53BP1, BRCA1, NBS1, and ATR (Fig. 5K). Taken together, these data suggest that CXCR4/STAT3/Slug axis is crucial for IR resistance in NSCLC cells.

\section{Combination treatment of irradiation and AMD3100 reduced tumor growth rate}

Next, we evaluated the therapeutic effect of combination of IR (10 Gy) and AMD3100 in vivo. Tumor-bearing mice were infused with AMD3100 starting at -1 day before IR. AMD3100 has no significant effect on the growth of non-irradiated tumor (Fig. 6A). IR alone or AMD3100 and IR combination treatment reduced the tumor growth rate. However, AMD3100 and IR combination treatment further demonstrated a reduced tumor growth rate in contrast to irradiation alone $(P<0.05)$ (Fig. 6A). TDT in control and AMD3100 alone were similar, as 3.78 and 3.70 day, respectively. TDT in irradiation alone and combination treatment is 6.19 and 7.20 day, respectively. IR treatment showed approximately 
A)

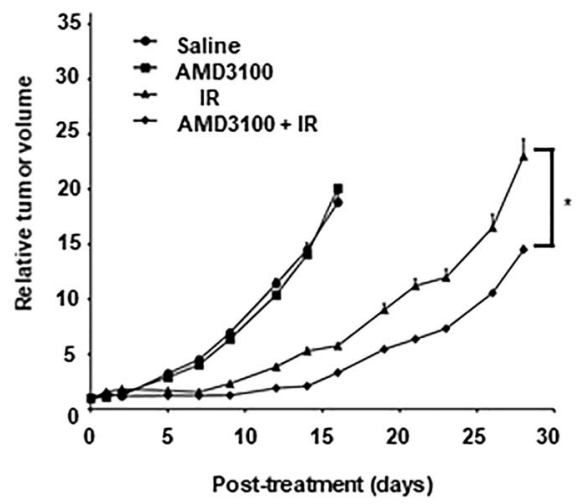

C)

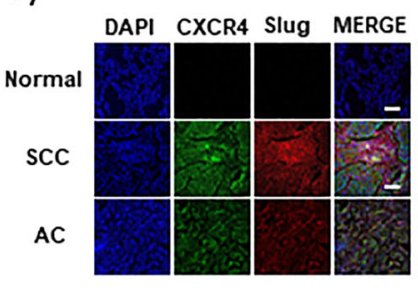

p-STAT3

(Y705) MERGE
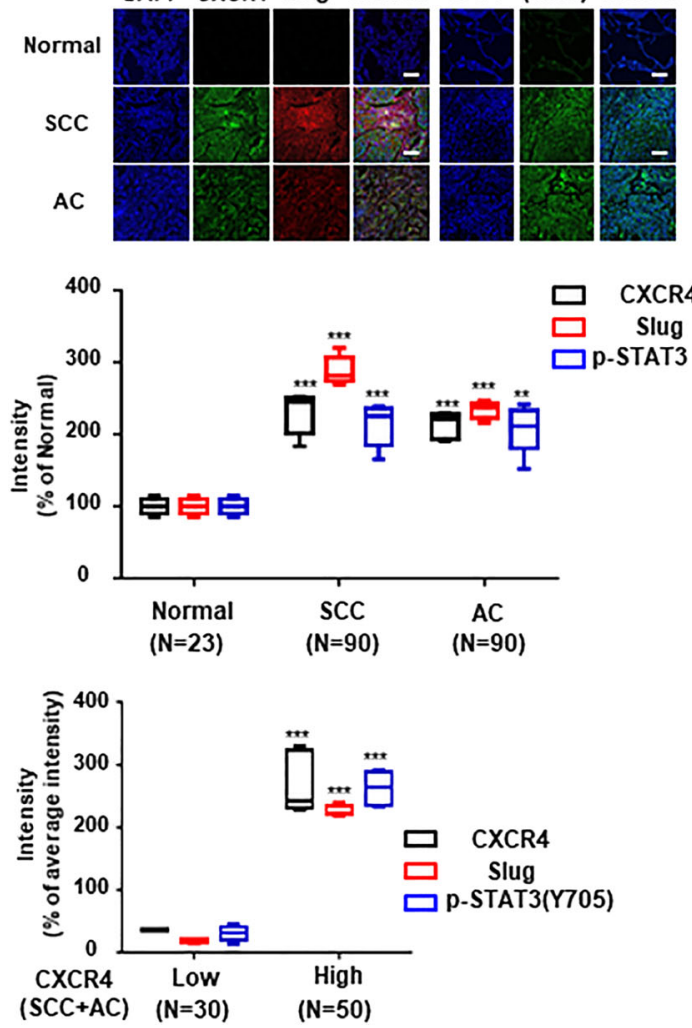

B)

Saline

IR

DAPI CXCR4 Slug MERGE DAPI (Y705) MERGE
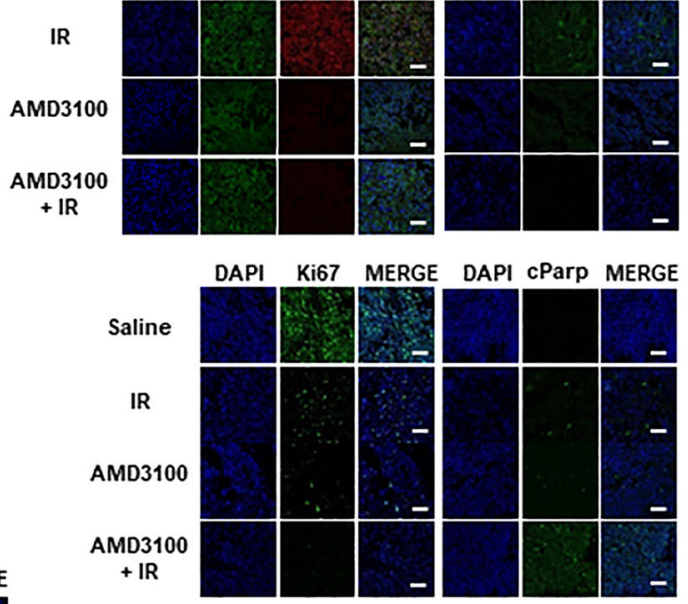

D)
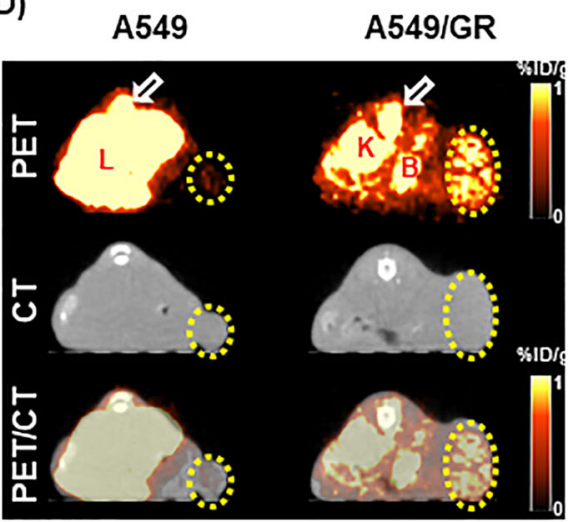

Fig. 6 Treatment of CXCR4 inhibitor enhances IR sensitivity in vivo. A AMD3100 (5 mg/kg) was released from the alzet pump implanted beneath the skin on the top of the neck of mice. IR (10 Gy) was focally exposed to the tumor when it reached to volume of $100 \mathrm{~mm}^{3}$. Detailed experimental procedure was described in the "Materials and methods" section. B Representative illustration of IHC of xenograft tissues using antibodies shown in the Figure. $\mathbf{C I H C}$ of NSCLC patients tissue array using antibodies is indicated in the Figure. Representative picture of the staining (in the upper part) and quantification of the fluorescence intensity (middle and lower). Nuclei were stained with DAPI. SCC squamous cell carcinoma, AC adenocarcinoma. White bar $100 \mu \mathrm{m}$. D PET/CT imaging of CXCR4 expression in subcutaneous A549 or A549/GR tumor xenografts with [ ${ }^{64}$ Cu] AMD3100. Athymic mice bearing A549 or A549/GR xenografts on the right flank, were given $\sim 11.1 \mathrm{MBq}(300 \mu \mathrm{Ci})$ of ${ }^{64} \mathrm{Cu}$-labeled radiotracers via tail vein injection, and PET/CT images were acquired. The representative transaxial PET, CT, and PET/CT sections of A549 or A549/GR tumors (yellow circle) from a $\left[{ }^{64} \mathrm{Cu}\right] \mathrm{AMD} 3100$-injected mouse at $90 \mathrm{~min}$ postinjection. Specific accumulation of radioactivity in A549/GR over A549 is apparent. The bone marrow (arrow), liver, and kidney uptake of $\left[{ }^{64} \mathrm{Cu}\right] \mathrm{AMD} 3100$ was also visualized. L liver, K kidney, B bladder. ${ }^{*} P<0.05$. 
1.6-times increased TDT, compared to control or AMD3100 alone. Combination (AMD3100 + IR) treatment showed 1.16-fold increased TDT, compared to IR alone. IHC of xenograft tissue demonstrated that AMD3100 and the combination of AMD3100 and IR significantly suppressed p-STAT3 and Slug in comparison to saline or IR alone sample (Fig. 6B, upper panel). Ki67 expression was dominantly suppressed in IR, AMD3100, whereas a combination of IR and AMD3100 samples. Meanwhile, cParp expression was significantly expressed through a combination of IR and AMD3100 sample, indicating the possible therapeutic enhancement of xenograft tumor (Fig. 6B, lower panel). Additionally, IHC of tissue microarray revealed that expression levels of CXCR4, p-STAT3, and Slug are much higher in NSCLC tissues than in normal tissues (Fig. 6C, upper and middle panels). Importantly, the expression of CXCR4 had a positive correlation with the expression of p-STAT3 and Slug within NSCLC tissues, thus indicating the possibility that CXCR4/STAT3/Slug pathway might be crucial for therapeutic resistance, including IR in NSCLC (Fig. 6C, lower panel).

Finally, to investigate the specific accumulation of ADM3100 on the CXCR4+ tumor cells, we radiolabeled it with ${ }^{64} \mathrm{Cu}$. The specific radioactivity of the $\left[{ }^{64} \mathrm{Cu}\right]$ AMD3100 after purification was typically $117 \mathrm{GBq} / \mu \mathrm{mol}$ $(3.17 \mathrm{Ci} / \mu \mathrm{mol})$, with radiochemical purity of $>98 \%$ as determined by radio-ITLC. In A549 and A549-GR cells, the uptake of $\left[{ }^{64} \mathrm{Cu}\right] \mathrm{AMD} 3100$ was $2.01 \pm 0.42 \% \mathrm{AD}$ and $0.82 \pm 0.20 \% \mathrm{AD}$, respectively. Furthermore, receptor quantification analysis revealed that A549 and A549/GR expressed $1549.8 \pm 378.0$ and $3817.8 \pm 793.8$ receptors per cell, respectively, accounting for differences in $\left[{ }^{64} \mathrm{Cu}\right]$ AMD3100 binding. The 90-min PET/CT images in the lung tumor model showed selective accumulation of activity in A549/GR tumor compared with A549 tumor model. The transaxial images at 90 min showed significant uptake in the liver, kidney, bladder, and bone marrow (Fig. 6B). The data indicate that cell surface CXCR4 can be considered as a theragnostic target in CXCR4 high NSCLC cells.

\section{Discussion}

Evidence indicates that despite availability of drastic interventions such as chemotherapy and radiotherapy certain cancer cells have been proven to cause formation of tumor bed and lead to poor prognosis. These cells have been established to have stem-like features. In the previous study, we have shown that CXCR4 is a functional CSC marker in NSCLC cells ${ }^{13}$. Since therapeutic resistance is whole mark of CSCs, we initially investigated the role of CXCR4 in the resistance to chemotherapeutic drugs and IR and found that there was no difference on drug resistance between CXCR4+ and
CXCR4 - cells, however we observed that A549/GR cells have much higher potential of resistance to $\mathrm{IR}^{13}$. In this study, we brought forward the role of CXCR4 in the IR resistance of NSCLC cells. Gefitinib resistant A549/GR cells showed higher clonogenic survival activity and led to more rapid resolution of $\gamma-\mathrm{H} 2 \mathrm{AX}$ foci formation upon IR treatment rather than A549 parent cells. Via the gain and loss of function study, we ascertain that CXCR4 signaling is crucial for the resistance of NSCLC cells towards IR. In addition, we also found that STAT3 is critically involved in the CXCR4-mediated resistance of IR in NSCLC cells. Finally, microarray analysis of NSCLC cells revealed that Slug is a downstream effector molecule of CXCR4/STAT3 signaling in the IR resistance of NSCLC cells. Therefore, we conclude that CXCR4/STAT3/Slug signaling axis has functional role in the IR resistance as well as maintenance of stemness in NSCLC cells.

Many studies have shown that CXCR4 is one of the markers for cancer stem cells in various types of cancer $^{12,19,20}$. Our and others' research also discovered that high level of CXCR4 expression is also found in stem-like lung cancer cells ${ }^{13,20}$. Since CSCs are well known as the causative cells of therapeutic resistance, we examined whether the crucialness of CXCR4 signaling for drug and IR resistance towards NSCLC cells. Using drug resistant A549/GR cells, we found the CXCR4 signaling is deeply involved in the resistance to IR, but not in drug resistance. Through knockdown and overexpression studies of CXCR4 in NSCLC cells, we deduced the important role of CXCR4 signaling in IR resistance of NSCLC cells. In line with our results, some reports have revealed that CXCR4 is a CSC marker and at the same time, an IR resistant marker $^{21-23}$. Therefore, as far as we know, this is the first evidence suggesting the CXCR4 can be a good target for enhancing radiosensitivity of NSCLC.

In the previous study, we found that CXCR4 preferentially signaled to STAT3 for the maintenance of stemness in NSCLC cells ${ }^{13}$. Several reports have shown that STAT3 signaling is involved in radiotherapeutic and chemotherapeutic sensitivity in NSCLC ${ }^{24-26}$. Therefore, in this study, we investigated whether STAT3 affects IR resistance of NSCLC cells or not. siRNA-mediated knockdown and pharmacologic inhibition of STAT3 indicated that CXCR4-mediated activation of STAT3 is crucial for IR resistance in NSCLC cells. Interestingly, phosphorylation of STAT3 at the tyrosine 705 (pYSTAT3) but not at the serine 727 sites (pS-STAT3) seemed to play an important role for IR resistance in NSCLC cells. Overall, our results and others suggest that STAT3 is a good target to increase radiotherapy effect of NSCLC.

In the present work, we found that the transcription factor Slug is deeply involved in the IR resistance of 
NSCLC cells as a downstream effector molecule for CXCR4/STAT3 signaling. The fact that Slug is involved in IR resistance has been studied in various types of cancers $^{27-32}$. As a transcriptional repressor, Slug protects cells by inhibiting the expression of puma, a Bcl-2 family proapoptotic protein, from p53-mediated apoptosis caused by DNA damage such as IR exposure ${ }^{30,33}$. In addition, recent report have shown that deficient of Slug leads to accumulation of DNA damage by impairing the recruitment of RAD51 at the damaged site ${ }^{34}$. Therefore, it is highly probable that CXCR4/STAT3 signaling augments the expression of Slug which suppresses puma expression and enhances the resolution of DNA damage by IR in NSCLC cells. Although the precise molecular intricacies of these effects in tumor could be more complex and involve cooperative functioning of other molecules and could partly be related to IR resistance, our findings definitely suggest that Slug is a critical determinant of CXCR4mediated IR resistance in these cells. Although we have focused on these phenomena in lung cancer, our findings can possibly be extended to other cancers to represent a common, yet underestimated, mechanism that could make cancer cells difficult to eradicate.

Summing up, this study observes that CXCR4/STAT3/ slug signaling is critical in maintaining IR resistance of NSCLC cells, and offers the possibility of targeting CXCR4 signaling in suppressing and eliminating CSCs of NSCLC by IR. Therefore, blockade of CXCR4/STAT3 pathway could be a promising approach for the efficient sensitizing NSCLC cells to IR, hence being useful for the radiotherapy of this devastating cancer.

\section{Acknowledgements}

This study was supported by a grant of the Korea Institute of Radiological and Medical Sciences (KIRAMS), funded by Ministry of Science and ICT (MSIT), Republic of Korea. (No. 50531-2020).

\section{Author details}

${ }^{1}$ Radiation Therapeutics Development Team, Division of Radiation Cancer Science, Korea Institute of Radiological and Medical Sciences, Seoul, Korea. ${ }^{2}$ School of Biomedical Science, Korea University, Seoul, Korea. ${ }^{3}$ Department of Life Sciences, Korea University, Seoul, Korea. ${ }^{4}$ Division of RI Application, Korea Institute of Radiological and Medical Sciences, Seoul, Korea. ${ }^{5}$ Department of Biochemistry, School of Medicine, Daegu Catholic University, 33, 17-gil, Duryugongwon-ro, Nam-gu, Daegu 42472, Korea

\section{Author contributions \\ J.Y.K., H.J.K., and M.J.P. conceived and designed experiments; J.Y.K. and H.J.K. developed the methods; J.Y.K., H.J.K., and C.W.J. performed data acquisition, analysis, and interpretation; T.S.L. performed animal experiments; E.H.K. and M.J. P. wrote, reviewed, and revised the manuscript; M.J.P. supervised the study overall. All authors have read and agreed to the published version of the manuscript.}

\section{Conflict of interest}

The authors declare that they have no conflict of interest.

\section{Publisher's note}

Springer Nature remains neutral with regard to jurisdictional claims in published maps and institutional affiliations.
Supplementary Information accompanies this paper at (https://doi.org/ 10.1038/s41419-020-03280-5).

Received: 6 May 2020 Revised: 31 October 2020 Accepted: 4 November 2020

Published online: 07 January 2021

\section{References}

1. Tu, Z. et al. CXCR4 is involved in CD133-induced EMT in non-small cell lung cancer. Int J. Oncol. 50, 505-514 (2017).

2. Garon, E. B. et al. Pembrolizumab for the treatment of non-small-cell lung cancer. N. Engl. J. Med. 372, 2018-2028 (2015).

3. Lemjabbar-Alaoui, H., Hassan, O. U., Yang, Y. W. \& Buchanan, P. Lung cancer: biology and treatment options. Biochim Biophys. Acta 1856, 189-210 (2015).

4. Templeton, A. K., Miyamoto, S., Babu, A., Munshi, A. \& Ramesh, R. Cancer stem cells: progress and challenges in lung cancer. Stem Cell Investig. 1, 9 (2014).

5. Lee, S. H. et al. SOX2 regulates self-renewal and tumorigenicity of stem-like cells of head and neck squamous cell carcinoma. Br. J. Cancer 111, 2122-2130 (2014).

6. Wang, X. Q. et al. Octamer 4 (Oct4) mediates chemotherapeutic drug resistance in liver cancer cells through a potential Oct4-AKT-ATP-binding cassette G2 pathway. Hepatology 52, 528-539 (2010).

7. Hardavella, G., George, R. \& Sethi, T. Lung cancer stem cells-characteristics, phenotype. Transl. Lung Cancer Res. 5, 272-279 (2016).

8. Phi, L. T. H. et al. Cancer stem cells (CSCS) in drug resistance and their therapeutic implications in cancer treatment. Stem Cells Int. 2018, 5416923 (2018).

9. Burger, J. A. \& Burkle, A. The CXCR4 chemokine receptor in acute and chronic leukaemia: a marrow homing receptor and potential therapeutic target. Br. J. Haematol. 137, 288-296 (2007).

10. Wang, Z. et al. Oncogenic roles and drug target of CXCR4/CXCL12 axis in lung cancer and cancer stem cell. Tumour Biol. 37, 8515-8528 (2016).

11. Kato, M., Kitayama, J., Kazama, S. \& Nagawa, H. Expression pattern of CXC chemokine receptor-4 is correlated with lymph node metastasis in human invasive ductal carcinoma. Breast Cancer Res. 5, R144-150. (2003).

12. Kim, J. et al. Chemokine receptor CXCR4 expression in colorectal cancer patients increases the risk for recurrence and for poor survival. J. Clin. Oncol. 23 2744-2753 (2005)

13. Jung, M. J. et al. Upregulation of CXCR4 is functionally crucial for maintenance of stemness in drug-resistant non-small cell lung cancer cells. Oncogene $\mathbf{3 2}$, 209-221 (2013).

14. Rho, J. K. et al. Epithelial to mesenchymal transition derived from repeated exposure to gefitinib determines the sensitivity to EGFR inhibitors in A549, a non-small cell lung cancer cell line. Lung Cancer 63, 219-226 (2009).

15. Kim, J. Y. et al. A simple Cu-64 production and its application of Cu-64 ATSM. Appl. Radiat. Isot. 67, 1190-1194 (2009).

16. Nimmagadda, S. et al. Molecular imaging of CXCR4 receptor expression in human cancer xenografts with $\left[{ }^{64} \mathrm{Cu}\right.$ AMD3100 positron emission tomography. Cancer Res. 70, 3935-3944 (2010).

17. Lee, J. et al. RGD peptide-conjugated multimodal NaGdF4:Yb3+/Er3+ nanophosphors for upconversion luminescence, $M R$, and PET imaging of tumor angiogenesis. J. Nucl. Med. 54, 96-103 (2013).

18. Kuo, L. J. \& Yang, L. X. Gamma-H2AX-a novel biomarker for DNA doublestrand breaks. In vivo 22, 305-309 (2008)..

19. Schimanski, C. C. et al. Dissemination of hepatocellular carcinoma is mediated via chemokine receptor CXCR4. Br. J. Cancer 95, 210-217 (2006).

20. Fareh, M. et al. The miR 302-367 cluster drastically affects self-renewal and infiltration properties of glioma-initiating cells through CXCR4 repression and consequent disruption of the SHH-GLI-NANOG network. Cell Death Differ. 19, 232-244 (2012).

21. Bertolini, G. et al. Highly tumorigenic lung cancer CD133+ cells display stemlike features and are spared by cisplatin treatment. Proc. Natl Acad. Sci. USA 106, 16281-16286 (2009).

22. Trautmann, F. et al. CXCR4 as biomarker for radioresistant cancer stem cells. Int J. Radiat. Biol. 90, 687-699 (2014).

23. Goffart, N. et al. CXCL12 mediates glioblastoma resistance to radiotherapy in the subventricular zone. NeuroOncology 19, 66-77 (2017) 
24. Yin, Z. J. et al. Overexpression of STAT3 potentiates growth, survival, and radioresistance of non-small-cell lung cancer (NSCLC) cells. J. Surg. Res. 171, 675-683 (2011).

25. Hsu, H. S. et al. Cucurbitacin I inhibits tumorigenic ability and enhances radiochemosensitivity in nonsmall cell lung cancer-derived CD133-positive cells. Cancer 117, 2970-2985 (2011).

26. You, S. et al. Disruption of STAT3 by niclosamide reverses radioresistance of human lung cancer. Mol. Cancer Ther. 13, 606-616 (2014).

27. Perez-Losada, J., Sanchez-Martin, M., Perez-Caro, M., Perez-Mancera, P. A. \& Sanchez-Garcia, I. The radioresistance biological function of the SCF/kit signaling pathway is mediated by the zinc-finger transcription factor Slug. Oncogene 22, 4205-4211 (2003).

28. Kurrey, N. K. et al. Snail and slug mediate radioresistance and chemoresistance by antagonizing p53-mediated apoptosis and acquiring a stem-like phenotype in ovarian cancer cells. Stem Cells 27, 2059-2068 (2009).
29. Arienti, C. et al. SLUG silencing increases radiosensitivity of melanoma cells in vitro. Cell Oncol. 36, 131-139 (2013).

30. Jiang, F., Zhou, L., Wei, C., Zhao, W. \& Yu, D. Slug inhibition increases radiosensitivity of oral squamous cell carcinoma cells by upregulating PUMA. Int. J. Oncol. 49, 709-719 (2016).

31. Yang, H., Zhang, G., Che, X. \& Yu, S. Slug inhibition increases radiosensitivity of nasopharyngeal carcinoma cell line C666-1. Exp. Ther. Med. 15, 3477-3482. (2018).

32. Lin, J. C., Tsai, J. T., Chao, T. Y., Ma, H. I. \& Liu, W. H. The STAT3/Slug axis enhances radiation-induced tumor invasion and cancer stem-like properties in radioresistant glioblastoma. Cancers 10, 12 (2018).

33. Wu, W. S. et al. Slug antagonizes p53-mediated apoptosis of hematopoietic progenitors by repressing puma. Cell 123, 641-653 (2005).

34. Gross, K. M. et al. Loss of slug compromises DNA damage repair and accelerates stem cell aging in mammary epithelium. Cell Rep. 28, 394-407 (2019). 